International Journal of Modern Physics C (C) World Scientific Publishing Company

\title{
Comparison between isothermal collision-streaming and finite-difference lattice Boltzmann models
}

\author{
G. Negro \\ Dipartimento di Fisica, Università degli Studi di Bari and INFN, \\ Sezione di Bari, via Amendola 173, Bari, I-70126, Italy \\ S. Busuioc \\ Department of Physics, West University of Timişoara, \\ Bd. Vasile Pârvan 4, 300223 Timişoara, Romania \\ V. E. Ambruş \\ Department of Physics, West University of Timişoara, \\ Bd. Vasile Pârvan 4, 300223 Timişoara, Romania \\ G. Gonnella \\ Dipartimento di Fisica, Università degli Studi di Bari and INFN, \\ Sezione di Bari, via Amendola 173, Bari, I-70126, Italy \\ A. Lamura \\ Istituto Applicazioni Calcolo, CNR, \\ Via Amendola 122/D, I-70126 Bari, Italy \\ V. Sofonea \\ Center for Fundamental and Advanced Technical Research, Romanian Academy, \\ Bd. Mihai Viteazul 24, 300223 Timişoara, Romania \\ Received Day Month Year \\ Revised Day Month Year
}

\begin{abstract}
We present here a comparison between collision-streaming and finite-difference lattice Boltzmann (LB) models. This study provides a derivation of useful formulae which help one to properly compare the simulation results obtained with both LB models. We consider three physical problems: the shock wave propagation, the damping of shear waves, and the decay of Taylor-Green vortices, often used as benchmark tests. Despite the different mathematical and computational complexity of the two methods, we show how the physical results can be related to obtain relevant quantities.
\end{abstract}

Keywords: Lattice Boltzmann Models; Finite-Difference; Collision-Streaming; Viscosity PACS Nos.: 


\section{Introduction}

Since more than 3 decades ago, the use of lattice Boltzmann (LB) models to address hydrodynamic problems has widely expanded because of the parallel nature of their basic algorithm, as well as of their capability to easily handle interparticle interactions and boundary conditions $12 \mid 3$. A characteristic feature of the LB models is the polynomial expansion 4 of the single-particle equilibrium distribution function $f^{e q}(\boldsymbol{x}, \boldsymbol{v}, t)$ up to a certain order $N$ with respect to the fluid velocity $\boldsymbol{u} \equiv \boldsymbol{u}(\boldsymbol{x}, t)$. A rigorous way to do this, is projecting the equilibrium distribution function on a set of orthogonal polynomials. In addition, the use of Hermite polynomials as the expansion basis has the unique feature that the expansion coefficients correspond precisely to the velocity moments 4 up to a given degree. The polynomial expansion and the application of the Gauss quadrature theorem allows one to compute the moments of $f^{e q}(\boldsymbol{x}, \boldsymbol{v}, t)$, as well as of the distribution function $f(\boldsymbol{x}, \boldsymbol{v}, t)$, which appear in the Boltzmann equation, by summation over a discrete velocity set $\boldsymbol{v}_{k}$, $1 \leq k \leq K ! 1|2| 3 \mid 4$

In the widely used collision-streaming (CS) lattice Boltzmann models, the velocity space is discretized so that the velocity vectors of the fluid particles leaving a node of the lattice are oriented towards the neighboring nodes.1213 Such models are also called on-lattice models. Alternatively, in the finite-difference (FD) lattice Boltzmann models $5\left[6\right.$, the velocity vectors $\boldsymbol{v}_{k}$ are obtained using the Gauss-Hermite quadrature method in the velocity space. These vectors are generally off-lattice and their Cartesian components are expressed as irrational numbers, namely the roots of the Hermite polynomials. For this reason, in these models the distribution functions are evolved using an appropriate finite-difference scheme. $617 / 8$

To the best of our knowledge, no comparison is available in the literature between the two schemes to properly match physical quantities in terms of the model parameters. In this study, we aim to provide the tools necessary to perform such comparisons, which may be used to compare, for example, past results obtained for nonideal fluids using various LB models. Indeed, in the past both CS 910 and FD $6[7|11| 12$ lattice Boltzmann models were used to study liquid-vapor systems. A comparison between the two methods lacked and no attempt was done so far to have a unified framework to map models onto each other.

The outline of this paper is as follows. In Sec. 2, we briefly describe the CS and the FD lattice Boltzmann models here considered. In Sec. 3 we derive the way enabling the conversion between the non-dimensionalization procedures currently used in these models. This ensures the simulation of the same physical system with the two models. In Sec. 4 we compare the two LB models (CS and FD) by considering simple problems involving an ideal fluid under the assumption of isothermal conditions. Our conclusions are summarised in Sec. 5 . 


\section{Lattice Boltzmann models}

When the Bhatnagar-Gross-Krook (BGK) collision term is used in an isothermal LB model, the moments of the distribution function $f(\boldsymbol{x}, \boldsymbol{v}, t)$ up to order $N=2$ are needed in order to get the evolution equations of the macroscopic fields at the incompressible Navier - Stokes level 1/21341314. The minimum number of the velocity vectors in the two-dimensional $(D=2)$ isothermal LB model based on the full-range Gauss-Hermite quadrature ensuring all the moments of $f(\boldsymbol{x}, \boldsymbol{v}, t)$ up to order $N=2$ is $\left.K=(N+1)^{D}=9 \underline{4}[15] 16\right] 17$

As usual in the current LB models involving the BGK collision term, $\frac{4}{4}$ the nondimensionalized form of the evolution equation of the functions $f_{k} \equiv f\left(\boldsymbol{x}, \boldsymbol{v}_{k}, t\right)$ for the force-free flow of a single-component fluid is

$$
\partial_{t} f_{k}+\boldsymbol{v}_{k} \cdot \nabla f_{k}=-\frac{1}{\tau}\left[f_{k}-f_{k}^{e q}\right],
$$

where $\tau$ is the non-dimensionalized value of the relaxation time. For simplicity, in this paper the value of $\tau$ is assumed to be constant. The details of the nondimensionalization procedure used in the FD and the CS lattice Boltzmann models are discussed in Section 3 below. The equilibrium single-particle distribution function, expanded up to order $N=2$, is given by:

$$
f_{k}^{e q}=w_{k} \rho\left\{1+\boldsymbol{v}_{k} \cdot \boldsymbol{u}+\frac{1}{2}\left[\left(\boldsymbol{v}_{k} \cdot \boldsymbol{u}\right)^{2}-u^{2}\right]\right\}
$$

After the aforementioned discretization of the velocity space, the macroscopic quantities, namely the fluid density $\rho$ and momentum density $\rho \boldsymbol{u}$, are computed as

$$
\left(\begin{array}{c}
\rho \\
\rho \boldsymbol{u}
\end{array}\right)=\sum_{k} f_{k}\left(\begin{array}{c}
1 \\
\boldsymbol{v}_{k}
\end{array}\right)
$$

When using the finite-difference LB model in this paper, the evolution equation (1) is solved by using the third order total variation diminishing (TVD) RungeKutta (RK-3) time stepping procedure, 18|19|20|21 together with the fifth-order weighted essentially non-oscillatory (WENO-5) scheme for the advection. 22|23|24

Using the Chapman-Enskog method, it can be shown that, when the fluid satisfies the Navier-Stokes equation, the non-dimensionalized value of the kinematic viscosity is given by

$$
\nu_{\mathrm{FD}}=\frac{\tau_{\mathrm{FD}} T}{m}
$$

where $\tau_{\mathrm{FD}}$ is the relaxation time non-dimensionalized with respect to the finite difference conventions discussed in Sec. 3, $T$ is the non-dimensionalized value of the local fluid temperature and $m$ is the non-dimensionalized value of the fluid particle mass.

In the collision-streaming LB models, the fluid particles collide in the lattice nodes and thereafter move in the time lapse $\delta t$ towards the neighboring nodes, with 
speed $c_{l}=\delta s / \delta t$ along the lattice links of spacing $\delta s$. The distribution functions follow the governing equation (in the BGK approximation):

$$
f_{k}\left(\boldsymbol{x}+\boldsymbol{v}_{k} \delta t, t+\delta t\right)-f_{k}(\boldsymbol{x}, t)=-\frac{\delta t}{\tau}\left[f_{k}(\boldsymbol{x}, t)-f_{k}^{\mathrm{eq}}(\boldsymbol{x}, t)\right],
$$

where $\left\{\boldsymbol{v}_{k}\right\}, 0 \leq k \leq K-1$, is the set of discrete velocities. The equilibrium functions $f_{k}^{\text {eq }}(\boldsymbol{x}, t)$ are given by a second order expansion of the Maxwell-Boltzmann distribution function with respect to the Hermite polynomials. $\frac{910}{10}$ In all the CS simulations, the non-dimensionalised values $(\delta s)_{\mathrm{LU}}=1$ and $(\delta t)_{\mathrm{LU}}=\sqrt{3} / 3$ were used to fix the non-vanishing Cartesian projections $v_{k ; \alpha}\left(\alpha=1, \ldots D,\left|v_{k ; \alpha}\right|=c_{l}\right)$ of the vectors $\boldsymbol{v}_{k}$, such that $c_{l}=\sqrt{3}$, as prescribed by the Gauss -Hermite quadrature on the $D 2 Q 9$ lattice 10

In the CS lattice Boltzmann model, the relaxation time $\tau$ controls the kinematic viscosity

$$
\nu_{\mathrm{LU}}=\left(\tau_{\mathrm{LU}}-\frac{\delta t}{2}\right)
$$

where the subscript LU stands for lattice units.

\section{Relations between non-dimensionalization conventions}

In order to relate the non-dimensional values for a quantity $\tilde{A}$ (the tilde indicates a dimensional quantity), obtained using two non-dimensionalization conventions $\left(A_{1}\right.$ and $A_{2}$ ), the following formula can be used 25 .

$$
A_{1}=A_{2} \frac{\tilde{A}_{\text {ref; } 2}}{\tilde{A}_{\text {ref } ; 1}}
$$

since $\tilde{A}=A_{1} \tilde{A}_{\text {ref } ; 1}=A_{2} \tilde{A}_{\text {ref } ; 2}$.

We wish to simulate the same fluid system using both the FD and the CS lattice Boltzmann models. Since the reference values used in these models may be different, but the computer simulations are usually performed using non-dimensionalized quantities, we need the conversion relations between the non-dimensionalized values of the physical quantities used to describe the fluid properties and the flow geometry within each model. In the sequel, we will use the subscripts FD and LU to denote the physical quantities in the FD and the CS models, respectively. We choose to use LU (which stands for "lattice units") since this notation is frequently encountered in the LB literature dealing with CS models.

Let us consider a fluid system whose characteristic length is $\widetilde{L}$, in which an ideal fluid with viscosity $\widetilde{\nu}$ is maintained at the constant temperature $\widetilde{T}_{0}=T_{0} \widetilde{T}_{\text {ref. }}$. In this paper, we assume that the reference temperature $\widetilde{T}_{\text {ref }}$, the reference pressure $\tilde{P}_{\text {ref }}$, the reference mass $\widetilde{m}_{\text {ref }}$, as well as the reference density $\tilde{\rho}_{\text {ref }}$ are identical in both the CS and the FD models. 
The reference speed in the two models is:

$$
\widetilde{c}_{\mathrm{ref} ; \mathrm{LU}}=\widetilde{c}_{\mathrm{ref} ; \mathrm{FD}}=\sqrt{\frac{\widetilde{K}_{B} \widetilde{T}_{\mathrm{ref}}}{\widetilde{m}_{\mathrm{ref}}}},
$$

where $\widetilde{K}_{B}$ is the Boltzmann constant, and $\widetilde{T}_{\text {ref }}$ is the reference temperature in both models.

Let the reference length in the FD approach be the system size $\widetilde{L}_{\mathrm{ref} ; \mathrm{FD}}=\widetilde{L}$, while in the CS approach, it is the lattice spacing. Considering that the CS simulation is performed on a lattice containing $N_{\mathrm{LU}}$ nodes along the characteristic length $\tilde{L}$, the reference length in the CS model is

$$
\widetilde{L}_{\mathrm{ref} ; \mathrm{LU}}=\frac{\widetilde{L}}{N_{\mathrm{LU}}}=\frac{\widetilde{L}_{\mathrm{ref} ; \mathrm{FD}}}{N_{\mathrm{LU}}} .
$$

The reference time in the FD approach is

$$
\widetilde{t}_{\text {ref;FD }}=\frac{\widetilde{L}}{\widetilde{c}_{\text {ref;FD }}} .
$$

The reference time in the $\mathrm{LU}$ approach is:

$$
\widetilde{t}_{\mathrm{ref} ; \mathrm{LU}}=\frac{\widetilde{L}}{N_{\mathrm{LU}} \widetilde{c}_{\mathrm{ref} ; \mathrm{LU}}}=\frac{\widetilde{t}_{\mathrm{ref} ; \mathrm{FD}}}{N_{\mathrm{LU}}} .
$$

In order to ensure that the same system is being simulated, the viscosity must be fixed. The reference viscosity in the FD approach is:

$$
\widetilde{\nu}_{\text {ref;FD }}=\frac{\widetilde{t}_{\text {ref;FD }} \widetilde{P}_{\text {ref }}}{\widetilde{\rho}_{\text {ref }}}=\frac{\widetilde{L} \widetilde{P}_{\text {ref }}}{\widetilde{c}_{\text {ref;FD }} \widetilde{\rho}_{\text {ref }}},
$$

being independent of the simulation details, such as number of nodes or time step, where $\widetilde{P}_{\text {ref }}$ is the reference pressure. The LU reference viscosity reads:

$$
\widetilde{\nu}_{\text {ref;LU }}=\frac{\widetilde{\nu}_{\text {ref;FD }}}{N_{\mathrm{LU}}} .
$$

Thus, the LU reference viscosity depends on the number of lattice nodes $N_{\mathrm{LU}}$.

This result, as well as the expression (6) of the non-dimensionalized viscosity value in the CS model, allows us to get the relation between the non-dimensionalized FD relaxation time $\tau_{F D}$ and the corresponding value of $\tau_{L U}$ :

$$
\tau_{\mathrm{LU}}=\nu_{\mathrm{LU}}+\frac{\delta t_{\mathrm{LU}}}{2}=N_{\mathrm{LU}} \tau_{\mathrm{FD}}+\frac{\sqrt{3}}{6},
$$

where the last term represents the numerical correction typical for collisionstreaming simulations.

\section{Numerical results}

We present here different standard physical-benchmark problems in order to compare the two models. In all simulations periodic boundary conditions were considered. 


\subsection{Shock Waves}

As a first test problem we consider the Cartesian shock problem.

The test consists of a one-dimensional Riemann problem: In an isothermal ideal gas at temperature $T$, the density is initialized as follows:

$$
\left\{\begin{array}{l}
\rho(x)=\rho_{\mathrm{L}} \quad \text { if } \quad x \leq x_{0} \\
\rho(x)=\rho_{\mathrm{R}} \quad \text { otherwise }
\end{array}\right.
$$

where $\rho_{\mathrm{L}}$ and $\rho_{\mathrm{R}}$ are the values of the density to the left and to the right of the initial discontinuity, which is located at $x=x_{0}$. Since in our simulation setup, the density is related to the pressure $P$ through $\rho=m P / T$, where $T$ is considered to be constant, we expect no contact discontinuity to appear in our simulation results. This can be seen by considering the Euler equations, reproduced below for the one-dimensional flow of an isothermal fluid:

$$
\partial_{t} \rho+\partial_{x}(\rho u)=0, \quad \partial_{t}(\rho u)+\partial_{x}\left(\rho u^{2}+P\right)=0 .
$$

Introducing the similarity variable

$$
\xi=\frac{x-x_{0}}{t}
$$

it can be seen that Eq. (16) reduces to:

$$
\frac{\partial u}{\partial \xi}=\frac{\xi-u}{\rho} \frac{\partial \rho}{\partial \xi}, \quad \frac{\partial P}{\partial \xi}=(\xi-u)^{2} \frac{\partial \rho}{\partial \xi} .
$$

Noting that $P=\rho c_{s}^{2}$, where $c_{s}=\sqrt{T / m}$ is the non-dimensionalised speed of sound in an isothermal fluid, the above equations are satisfied either when $\rho$ and $u$ are constant, or when

$$
u=\xi \pm c_{s} .
$$

The above solution corresponds to a rarefaction wave travelling to the left $(+)$ or to the right $(-)$. We note that the solution $u=\xi$ (corresponding to the contact discontinuity) does not appear in the case of isothermal flows.

Assuming that $\rho_{\mathrm{L}}>\rho_{\mathrm{R}}$, the rarefaction wave propagates to the left, in which case the velocity can be seen to increase linearly according to:

$$
u_{*}\left(\xi_{*}\right)=\xi_{*}+c_{s},
$$

where the $\operatorname{star}(*)$ is employed to indicate that the analysis is restricted to the rarefaction wave. From Eq. (20) it can be seen that the head of the rarefaction wave travels with constant velocity

$$
\xi_{r}=-c_{s} .
$$

The tail of the rarefaction wave corresponds to the value $\xi_{c}$ of the similarity variable, for which the velocity takes the constant value on the plateau, $u=u_{c}$ :

$$
\xi_{c}=c_{s}(\zeta-1), \quad \zeta=\frac{u_{c}}{c_{s}},
$$


where the dimensionless quantity $\zeta$ was introduced for future convenience. The value of $u_{c}$ will be determined further below.

Inserting Eq. (20) into Eq. (18) gives the solution

$$
\rho_{*}\left(\xi_{*}\right)=\rho_{\mathrm{L}} \exp \left(-\frac{\xi_{*}-\xi_{r}}{\xi_{r}}\right)=\rho_{\mathrm{L}} \exp \left[-\zeta \frac{u_{*}\left(\xi_{*}\right)}{u_{c}}\right] .
$$

It can be seen that the density on the central plateau, $\rho_{c}$, can be determined once $\zeta$ is known using the equation

$$
\rho_{c}=\rho_{\mathrm{L}} e^{-\zeta} \text {. }
$$

Let us now consider the Rankine-Hugoniot junction conditions for a discontinuity having the similarity variable $\xi_{s}$ :

$$
\rho_{+}\left(u_{+}-\xi_{s}\right)=\rho_{-}\left(u_{-}-\xi_{s}\right), \quad \rho_{+} u_{+}\left(u_{+}-\xi_{s}\right)+P_{+}=\rho_{-} u_{-}\left(u_{-}-\xi_{s}\right)+P_{-},
$$

where + and - denote the fluid properties to the right and to the left of the discontinuity, respectively. Specializing the above equations to the case of the shock front, where $\rho_{+}=\rho_{\mathrm{R}}$ and $u_{+}=0$, the following relations are obtained:

$$
\xi_{s}=\frac{\rho_{c} \zeta c_{s}}{\rho_{c}-\rho_{\mathrm{R}}}, \quad \rho_{c}-\zeta^{2} \frac{\rho_{c} \rho_{\mathrm{R}}}{\rho_{c}-\rho_{\mathrm{R}}}-\rho_{\mathrm{R}}=0 .
$$

Inserting $\rho_{c}$ from (24) in the above relations, the value of $\zeta$ can be found by solving the following nonlinear equation:

$$
2+\zeta^{2}-\frac{\rho_{\mathrm{L}}}{\rho_{\mathrm{R}}} e^{-\zeta}-\frac{\rho_{\mathrm{R}}}{\rho_{\mathrm{L}}} e^{\zeta}=0 .
$$

In order to obtain the full solution, the value of $\zeta$ must be inserted in Eqs. (22) and (24) to obtain the velocity $\xi_{c}$ of the tail of the rarefaction wave and the density $\rho_{c}$ of the central plateau. The velocity $\xi_{s}$ of the shock front can be obtained from Eq. (26):

$$
\xi_{s}=\frac{\zeta c_{s}}{1-\frac{\rho_{\mathrm{R}}}{\rho_{\mathrm{L}}} e^{\zeta}} .
$$

We now discuss our numerical results. We consider that the fluid temperature is the reference temperature, such that $T=T_{\mathrm{LU}}=T_{\mathrm{FD}}=1$. Hence the nondimensionalized sound speed in both LB models is $c_{s}=1$. In order to reduce the errors due to compressibility effects, we take $\rho_{\mathrm{L}}=1.1$ and $\rho_{\mathrm{R}}=0.9$, where the reference density is taken to be the average of $\rho_{\mathrm{L}}$ and $\rho_{\mathrm{R}}$. In this case, $\zeta \simeq 0.10035$ and the relevant finite difference quantities are given below:

$$
\xi_{r}=-1, \quad \xi_{c} \simeq-0.900, \quad u_{c}=\zeta \simeq 0.10035, \quad \rho_{c} \simeq 0.995, \quad \xi_{s} \simeq 1.051 .
$$

The discontinuity in density makes the simulation of shock waves propagation a good test for the numerical methods used. The initial density jump creates a density wave traveling from high density regions to lower density ones. We fixed the number of nodes at $N_{x}=N_{L U}=2048$ and considered two values of the relaxation time, namely $\tau_{\mathrm{FD}}=\left\{10^{-4}, 10^{-3}\right\}$, corresponding to $\tau_{\mathrm{LU}}=\{0.493,2.33\}$. In Fig. 1] 

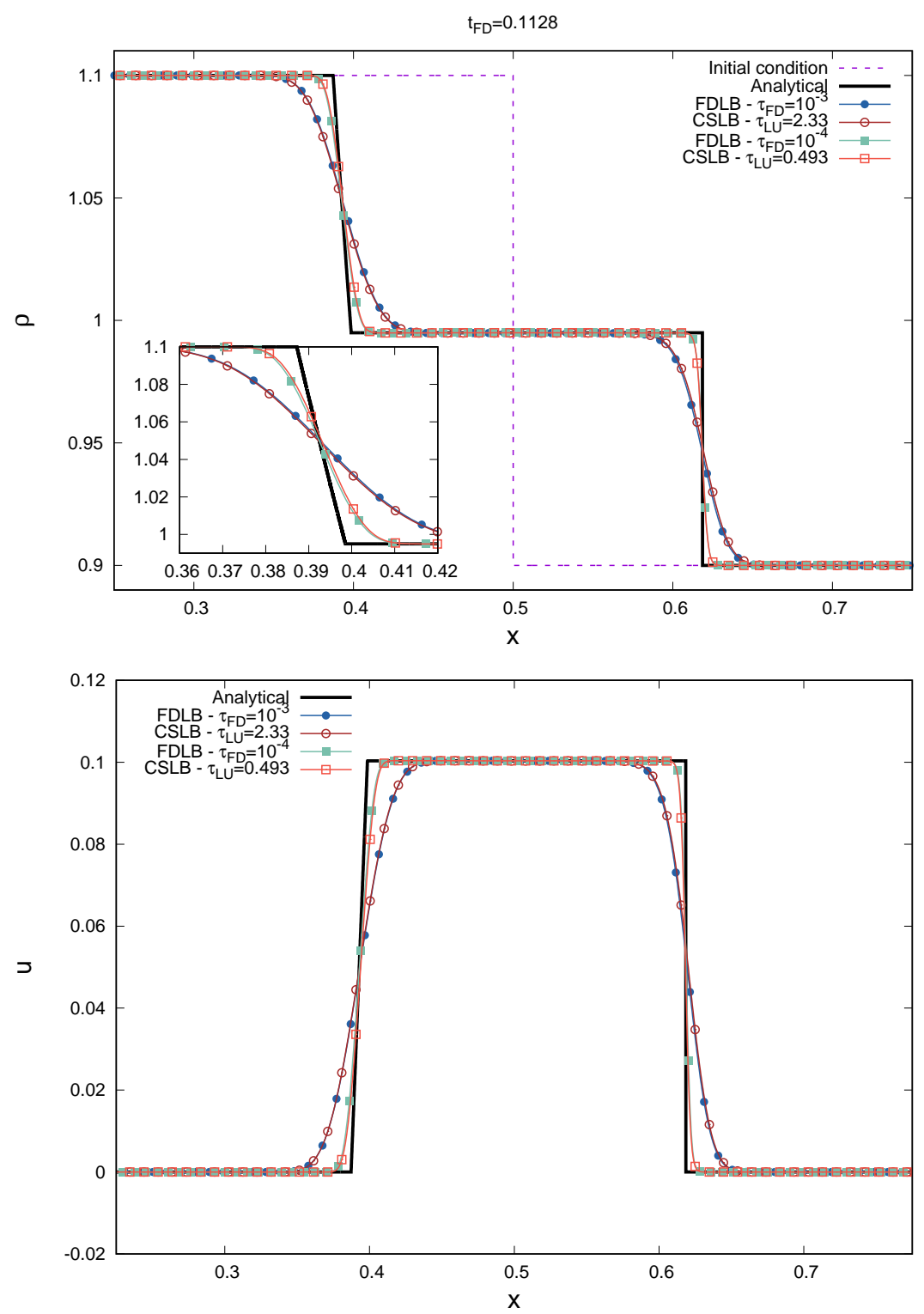

Fig. 1. Comparison of FDLB and CSLB results in the context of the Cartesian shock problem at the level of the density (upper panel) and velocity (lower panel) profiles, obtained at $t_{\mathrm{FD}} \simeq 0.1128$ for various values of the relaxation time. The inset shows the shock front.

the density and velocity profiles obtained with the two methods are represented at time $t_{\mathrm{FD}} \simeq 0.1128$ (attained after 400 iteration using CSLB), alongside the analytic solution for the inviscid case. The curves show good agreement between the two models for the considered values of viscosity. 
In the CSLB implementation, $(\delta t)_{\mathrm{LU}}=1 / \sqrt{3}$ corresponds to the time step $(\delta t)_{\mathrm{FD}}=1 / N \sqrt{3} \simeq 2.82 \times 10^{-4}$. The Courant-Friedrichs-Lewy number, CFL $=$ $c_{l} \delta t / \delta s$, is equal to one for this choice of parameters. In the FDLB implementation, the time step is bounded by the CFL condition CFL $\leq 1$, such that the maximum time step permitted is that employed in the CSLB implementation. The time step in the FDLB implementation is further restricted to obey $(\delta t)_{\mathrm{FD}}<\tau_{\mathrm{FD}}$, in order to prevent the collision term from becoming stiff. Thus, at $\tau_{\mathrm{FD}}=10^{-3}$, we performed the FDLB simulations using $(\delta t)_{\mathrm{FD}}=1 / N \sqrt{3}$, while at $\tau_{\mathrm{FD}}=10^{-4}$, the time step was decreased by a factor of $3,(\delta t)_{\mathrm{FD}}=1 / 3 N \sqrt{3} \simeq 9.40 \times 10^{-5}$, such that 1200 iterations were required to reach the state shown in Fig. 1. We note that the restriction $(\delta t)_{\mathrm{FD}}<\tau_{\mathrm{FD}}$ can be lifted, e.g., when implicit-explicit (IMEX) schemes are employed. 26

\subsection{Shear waves}

In order to compare numerical viscosity effects in the two models, we analyze in this subsection the evolution of shear waves. We consider waves of wavelength $\lambda=1$ in an ideal gas with density $\rho=1$ at temperature $T=1$.

In the simulations performed, the wave vector $\boldsymbol{k},|\boldsymbol{k}|=2 \pi / \lambda=2 \pi$, was aligned along the horizontal axis and its Cartesian components were $(2 \pi, 0)$.

Table 1. Apparent kinematic viscosity $\nu_{a p p}$, expressed using the FD adimensionalization, measured as a numerical fit of Eq. (32) in the context of the damping of shear waves.

\begin{tabular}{|ll|ll|ll|}
\hline & & \multicolumn{2}{|c|}{ CSLB } & \multicolumn{2}{c|}{ FDLB } \\
\hline$\tau_{\text {LU }}$ & $N_{x}$ & $\nu_{a p p}$ & Rel. err. & $\nu_{a p p}$ & Rel. err. \\
0.2986 & 20 & 0.0005073 & 0.0154 & 0.0005271 & 0.0542 \\
0.3036 & 30 & 0.0005032 & 0.0065 & 0.0005039 & 0.0078 \\
0.3086 & 40 & 0.0005017 & 0.0034 & 0.0005009 & 0.0018 \\
0.3136 & 50 & 0.0005015 & 0.0021 & 0.0005003 & 0.0006 \\
0.3186 & 60 & 0.0005006 & 0.0013 & 0.0005001 & 0.0002 \\
\hline
\end{tabular}

Let $\boldsymbol{u}(\boldsymbol{x}, t)$ be the fluid velocity vector. In both series of simulations, the velocity field was initialized according to:

$$
\begin{aligned}
& u_{x}(\boldsymbol{x}, 0)=0, \\
& u_{y}(\boldsymbol{x}, 0)=U \sin (\boldsymbol{k} \cdot \boldsymbol{x}),
\end{aligned}
$$

with $U=0.01$. When the fluid is not too far from the equilibrium (i.e., when the relaxation time is small enough), the fluid evolves according to the Navier-Stokes equations. In the setup of the shear waves problem, we have $u_{x}(\boldsymbol{x}, t)=0$ and there is no spatial variation of the velocity vector along the $y$ direction. Under these circumstances and assuming that the fluid is isothermal and incompressible, the Navier-Stokes equations reduce to:

$$
\partial_{t} u_{y}(\boldsymbol{x}, t)-\nu_{0} \partial_{x}^{2} u_{y}(\boldsymbol{x}, t)=0 .
$$




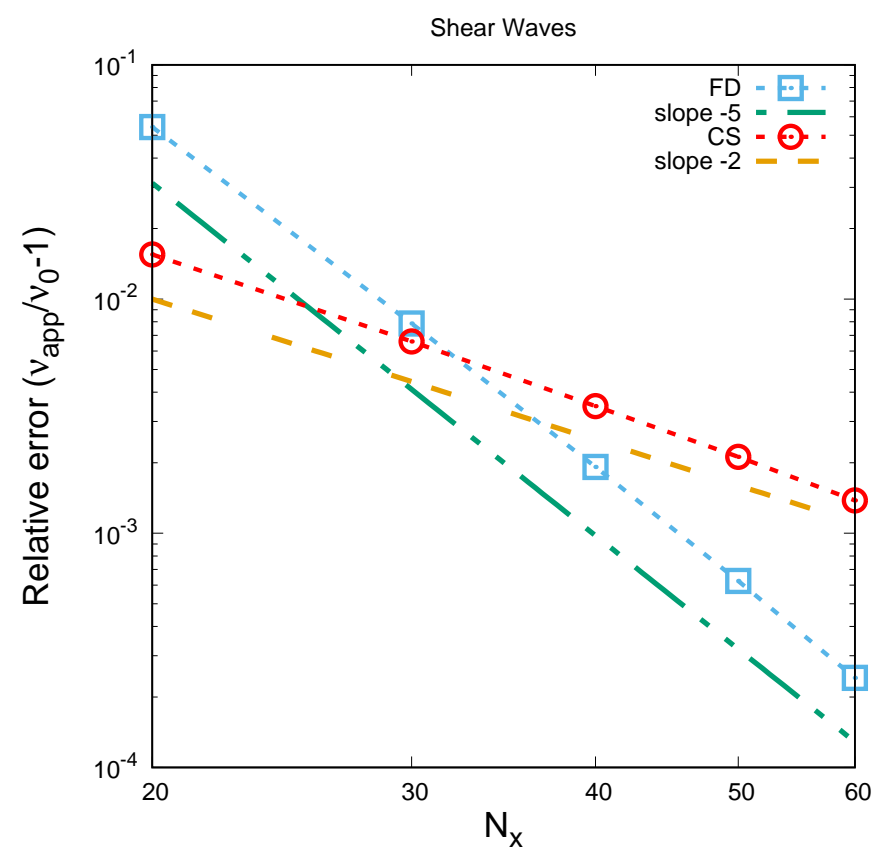

Fig. 2. Relative error of the measured kinematic viscosity $\nu_{a p p}$ expressed with respect to the expected analytic value $\nu_{0}$, extracted from the numerical simulations of the decaying shear waves problem, expressed with respect to the number of nodes $N_{x}$. A second second order convergence is recovered for the CSLB method, while for the FDLB method, the convergence is of fifth order.

Assuming that for $t>0, u_{y}(\boldsymbol{x}, t)=\widetilde{u}(t) \sin (\boldsymbol{k} \cdot \boldsymbol{x})$, the solution is:

$$
\widetilde{u}(t)=U e^{-k^{2} \nu_{0} t}
$$

where $\nu_{0}$ is the analytic kinematic viscosity.

We fixed the value of the kinematic viscosity in FD units at $\nu_{0 ; \mathrm{FD}}=\tau_{\mathrm{FD}}=$ $5 \times 10^{-4}$, and the simulations were performed for various values of $N_{x}=N_{L U}$. For a given value of $\nu_{\mathrm{FD}}$ and number of lattice nodes $N_{x}$, we used Eq. (14) to obtain the corresponding value of $\tau_{\mathrm{LU}}$, in order to simulate the exact same system with the CS and FD models. For the FD model we used a time step of $(\delta t)_{\mathrm{FD}}=5 \times 10^{-4}$ and lattice spacing $(\delta s)_{\mathrm{FD}}=1 / N_{x}$. In the CS model, the time step $(\delta t)_{\mathrm{LU}}=1 / \sqrt{3}$ corresponds to $(\delta t)_{\mathrm{FD}}=1 / N_{x} \sqrt{3} \simeq 5 \times 10^{-4} \times\left(1155 / N_{x}\right)$, which for $20 \leq N_{x} \leq$ 60 is around 20 to 60 times larger than the time step employed in the FDLB implementation.

In order to perform a quantitative analysis, a numerical fit of Eq. (32) was performed, which allows the parameter $\nu_{\text {app }}$ to be extracted. The measured values of $\nu_{a p p}$ are reported in Table 1 with the corresponding relative error. The latter is plotted in Fig. 2, showing a second order convergence for CS and a fifth order one for FD and confirming the expected numerical accuracy of the used models. It is worth 


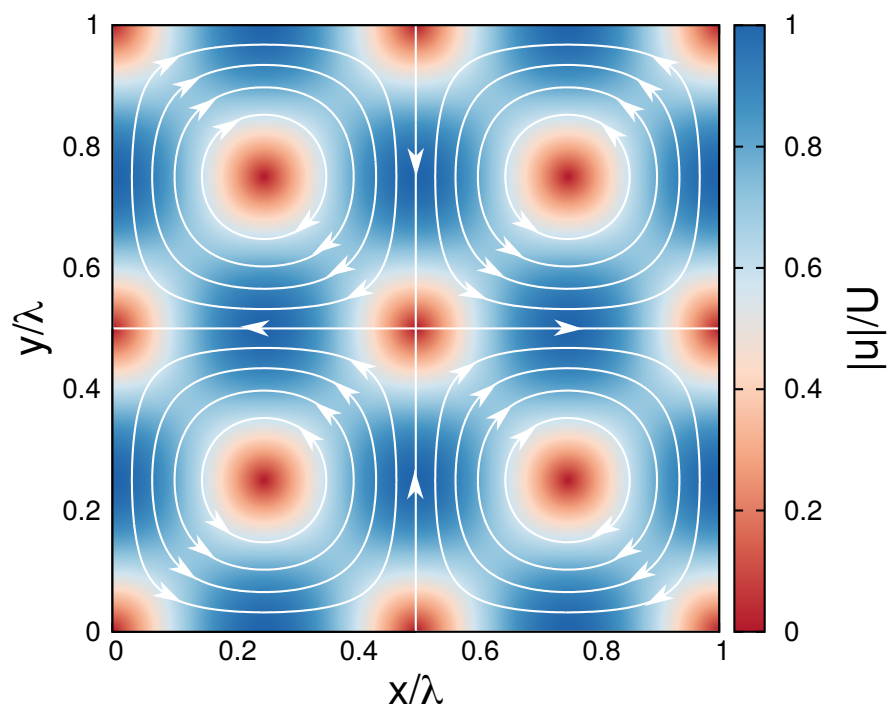

Fig. 3. Initial structure of a Taylor-Green vortex flow. Contour plot of the velocity field module is shown, with superimposed velocity stream lines.

noting that at $N_{x}=20$, the relative error when the CSLB method is employed is roughly 3.5 times smaller than the one corresponding to the FDLB method. The relative error of the FDLB results becomes smaller than that corresponding to the CSLB method when $N_{x} \gtrsim 30$.

\subsection{Taylor-Green vortices}

A parallel check for the kinematic viscosity can be performed by analyzing the damping of 2D Taylor-Green vortices. The system is initialized as follows:

$$
\begin{aligned}
& u_{x}=U \sin (k x) \cos (k y), \\
& u_{y}=-U \cos (k x) \sin (k y),
\end{aligned}
$$

where the amplitude is $U=0.01$ and the wave vector is $\boldsymbol{k}=(k, k)$, with $k=2 \pi / \lambda=$ $2 \pi$.

Similarly to the shear wave case, if we assume that for $t>0$, Eq. (33) holds with the amplitude $U$ replaced by $\widetilde{u}(t)$, then

$$
\widetilde{u}(t)=U e^{-2 k^{2} \nu_{0} t}
$$

Fig. 3 shows the initial structure of a Taylor-Green vortex flow. The flow maintains the same structure while decaying exponentially.

We fixed again the value of the kinematic viscosity in $\mathrm{FD}$ units at $\nu_{0 ; F D}=$ $5 \times 10^{-4}$, and the simulations were performed on square lattices having various number of nodes $N_{x}=N_{y}=N_{L U}=N$. The measured values of $\nu_{a p p}$, obtained by numerically fitting the simulation results with Eq. (34), are reported in Table 2 


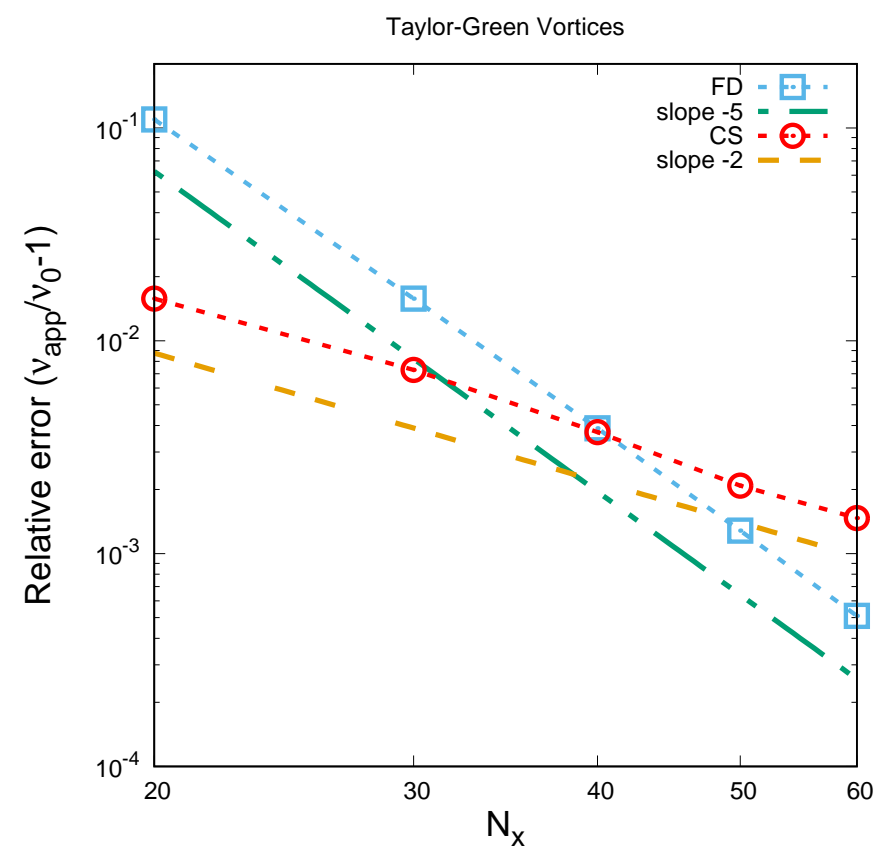

Fig. 4. Relative error of the measured kinematic viscosity $\nu_{a p p}$ expressed with respect to the expected analytic value $\nu_{0}$, extracted from the numerical simulations of the decaying TaylorGreen vortices, expressed with respect to the number of nodes $N_{x}$. A second order convergence is recovered for the CSLB method, while for the FDLB method, the convergence is of fifth order.

alongside the corresponding relative error. The latter is plotted in Fig. 4 showing again a second order convergence for CS and a fifth order one for FD with respect to the number of nodes. At $N=20$, the relative error obtained using the CSLB model is about 7 times smaller than the one corresponding to the FDLB results. The relative error of the FDLB results becomes smaller than the corresponding CSLB error when $N_{x} \gtrsim 40$.

Table 2. Apparent kinematic viscosity $\nu_{a p p}$, expressed using the FD adimensionalization, measured as a numerical fit of Eq. (34) in the context of the damping of the Taylor-Green vortices.

\begin{tabular}{|ll|ll|ll|}
\hline & & \multicolumn{2}{|c|}{ CSLB } & \multicolumn{2}{c|}{ FDLB } \\
\hline$\tau_{\text {LU }}$ & $N_{x}$ & $\nu_{a p p}$ & Rel. err. & $\nu_{a p p}$ & Rel. err. \\
0.2986 & 20 & 0.0005078 & 0.0157 & 0.0005548 & 0.1096 \\
0.3036 & 30 & 0.0005036 & 0.0072 & 0.0005079 & 0.0158 \\
0.3086 & 40 & 0.0005018 & 0.0037 & 0.0005019 & 0.0039 \\
0.3136 & 50 & 0.0005010 & 0.0020 & 0.0005006 & 0.0013 \\
0.3186 & 60 & 0.0005007 & 0.0014 & 0.0005002 & 0.0005 \\
\hline
\end{tabular}




\section{Conclusions}

We presented a comparison between lattice Boltzmann models implemented using the collision-streaming (CSLB) and finite-difference (FDLB) approaches. By matching the physical parameters such as the kinematic viscosity and system size, we showed how the results obtained using the two implementations can be related with each other, despite the different mathematical and computational complexity of these two methods.

We considered three different problems, namely the propagation of shock waves, the damping of shear waves and the damping of the Taylor-Green vortices. A good agreement between the two models was observed when the simulation parameters were chosen to correspond to the same physical quantities.

By providing the tools necessary to control the relevant physical quantities within the FD and CS approaches, our study confirms that is possible to simulate the same physical system using these two approaches, thus paving the way to address in the future fluid systems for wider ranges of parameters. This will be useful, e.g., in future simulations of nonideal fluids, since using both the CS and FD approaches can allow wider ranges of the parameter space to be explored.

\section{Acknowledgments}

V. E. Ambruş and S. Busuioc acknowledge funding from the Romanian Ministry of Research and Innovation, CCCDI-UEFISCDI, project number PN-III-P1-1.2PCCDI-2017-0371/VMS, within PNCDI III.

\section{References}

1. S. Chen and G. D. Doolen. Lattice Boltzmann method for fluid flows. Annual Review of Fluid Mechanics, 30(1):329-364, 1998.

2. T. Krüger, H. Kusumaatmaja, A. Kuzmin, O. Shardt, G. Silva, and E. M. Viggen. The lattice Boltzmann method: Principles and practice. Springer, Switzerland, 2017.

3. S. Succi. The Lattice Boltzmann Equation for Complex States of Flowing Matter. Oxford University Press, Oxford, 2018.

4. X. W. Shan, X. F. Yuan, and H. D. Chen. Kinetic theory representation of hydrodynamics: a way beyond the Navier-Stokes equation. J. Fluid. Mech., 550:413-441, 2006.

5. T. Biciuscă, A. Horga, and V. Sofonea. Simulation of liquid-vapour phase separation on GPUs using Lattice Boltzmann models with off-lattice velocity sets. Comptes Rendus Mécanique, 343:580-588, 2015.

6. V. Sofonea, T. Biciuşcă, S. Busuioc, V. E. Ambruș, G. Gonnella, and A. Lamura. Corner-transport-upwind lattice Boltzmann model for bubble cavitation. Phys. Rev. E, 97:023309, 2018.

7. A. Cristea, G. Gonnella, A. Lamura, and V. Sofonea. Finite-difference lattice Boltzmann model for liquid-vapor systems. Mathematics and Computers in Simulation, $72(2): 113-116,2006$.

8. A. Cristea, G. Gonnella, A. Lamura, and V. Sofonea. A lattice Boltzmann study of phase separation in liquid-vapor systems with gravity. Communications in Computational Physics, 7:350, 2009. 
9. G. Kähler, F. Bonelli, G. Gonnella, and A. Lamura. Cavitation inception of a van der Waals fluid at a sack-wall obstacle. Physics of Fluids, 27(12):123307, 2015.

10. A. Coclite, G. Gonnella, and A. Lamura. Pattern formation in liquid-vapor systems under periodic potential and shear. Phys. Rev. E, 89:063303, Jun 2014.

11. V. Sofonea, A. Lamura, G. Gonnella, and A. Cristea. Finite-difference lattice Boltzmann model with flux limiters for liquid-vapor systems. Phys. Rev. E, 70:046702, 2004.

12. G. Gonnella, A. Lamura, and V. Sofonea. Lattice Boltzmann simulation of thermal non ideal fluids. Phys. Rev. E, 76:036703, 2007.

13. Z.L. Guo and C. Shu. Lattice Boltzmann Method and its Applications in Engineering. World Scientific Publishing Co. Pte. Ltd., Singapore, 2013.

14. V. E. Ambruș and V. Sofonea. High-order thermal lattice Boltzmann models derived by means of Gauss quadrature in the spherical coordinate system. Phys. Rev. E, 86:016708, 2012.

15. P. Fede, V. Sofonea, R. Fournier, S. Blanco, O. Simonin, G. Lepoutère, and V. E. Ambrus, Lattice Boltzmann model for predicting the deposition of inertial particles transported by a turbulent flow. Int. J. Multiph. Flow, 76:187-197, 2015.

16. V. E. Ambruș and V. Sofonea. Lattice Boltzmann models based on half-range GaussHermite quadratures. J. Comput. Phys., 316:760-788, 2016.

17. B. Piaud, S. Blanco, R. Fournier, V. E. Ambrus, and V. Sofonea. Gauss quadratures - the keystone of lattice Boltzmann models. Int. J. Mod. Phys. C, 25:1340016, 2014.

18. S. Gottlieb and C.-W. Shu. Total variation diminishing Runge-Kutta schemes. Math. Comp., 67:73-85, 1998.

19. A. K. Henrick, T. D. Aslam, and J. M. Powers. Mapped weighted essentially nonoscillatory schemes: Achieving optimal order near critical points. J. Comput. Phys, 207:542-567, 2005.

20. C.-W. Shu and S. Osher. Efficient implementation of essentially non-oscillatory shockcapturing schemes. J. Comput. Phys., 77:439-471, 1988.

21. J. A. Trangenstein. Numerical solution of hyperbolic partial differential equations. Cambridge University Press, New York, 2007.

22. Y. Gan, A. Xu, G. Zhang, and Y. Li. Lattice Boltzmann study on Kelvin-Helmholtz instability: Roles of velocity and density gradients. Phys. Rev. E, 83:056704, 2011.

23. G. S. Jiang and C. W. Shu. Efficient implementation of weighted ENO schemes. J. Comput. Phys., 126:202-228, 1996.

24. S. Busuioc, V.E. Ambruş, T. Biciuşcă, and V. Sofonea. Two-dimensional off-lattice Boltzmann model for van der Waals fluids with variable temperature. Computers $\&$ Mathematics with Applications, 2019 (accepted for publication).

25. J.M. Yeomans. Mesoscale simulations: Lattice Boltzmann and particle algorithms. Physica A: Statistical Mechanics and its Applications, 369(1):159 - 184, 2006.

26. Y. Wang, Y. L. He, T. S. Zhao, G. H. Tang, and W. Q. Tao. Implicit-explicit finitedifference lattice Boltzmann method for compressible flows. Int. J. Mod. Phys. C, 18:1961-1983, 2007. 